\title{
Transgenic animals
}

\author{
BY KEVIN DOCHERTY \\ Department of Molecular and Cell Biology, University of Aberdeen, Marischal College, \\ Aberdeen $A B 91 A S$
}

The use of transgenic animals is becoming increasingly widespread in biomedical research. Applications range from studies on the cell-specificity and developmental timing of gene expression to the production of novel cell lines for use in research and for the production of novel therapeutic proteins. Gene knock-out experiments have now become common as one of the definitive methods for establishing the role of a particular gene product in a biochemical pathway or physiological process. The present review describes the techniques employed in the production of transgenic animals, and provides some examples of the uses to which transgenic animals have been put.

\section{THE TECHNIQUES}

\section{Production of transgenic animals by micro-injection of fertilized eggs}

The technique essentially involves removing fertilized eggs from the oviducts of mated females, injecting these eggs with the DNA of interest, and re-implanting the eggs in the oviduct of a pseudopregnant female, i.e. a female that has been mated with a vasectomized male. The fertilized eggs contain two pronuclei, one provided by the male and one by the female. A few picolitres of solution containing the DNA is injected into one of the pronuclei. In mice, the percentage of the manipulated eggs that survive to term is in the region of $30 \%$. However, this value varies with species and in some cases the efficiency can be very low $(<1 \%)$. Integration of the foreign DNA (transgene) is checked in offspring by Southern blot or polymerase-chain-reaction analysis of DNA extracted from a small section from the tail. The percentage of the offspring harbouring the transgene is also variable ranging from approximately 5 to $40 \%$. To determine whether the transgene has been integrated into germ cells, transgenic mice (founder generation) are mated with non-transgenic mice and the inheritance of the transgene followed through several generations. All the mice descended from a particular founder constitute a transgenic line.

\section{Embryonic stem cells}

One of the problems of producing transgenic mice by micro-injection of fertilized eggs is that there is little control over the way in which the DNA integrates into the genome. The foreign DNA appears to integrate randomly with multiple copies forming a tandem repeat. However, a much better control of integration can be achieved by micro-injecting the foreign DNA into embryonic stem cells, which are prepared from 3-d-old blastocysts. The blastocysts are removed from mice and cultured in a way that allows separation of the inner cell mass, i.e. the cells that will form the future embyro. These cells are plated out on a feeder layer of fibroblasts, under conditions that will allow them to grow and divide. When injected back into mice blastocysts these embryonic stem cells can participate in the formation of all tissues (Evans \& Kaufman, 1981). 
Foreign DNA can be introduced into embryonic stem cells by a variety of methods including DNA-mediated gene transfer (transfection), retroviral infection, electroporation, or micro-injection. This has the advantage that the transfected cells can be selected by including a selectable marker which confers antibiotic resistance.

\section{Homologous recombination and gene targeting}

Foreign DNA can be introduced into a specific location in the genome by homologous recombination (Smithies et al. 1984; Capecchi, 1989). Such gene targeting can be used to inactivate genes by disrupting the structure of the gene, or it can be used to replace an endogenous defective gene. Homologous recombination is a rare event and the success of the technique depends on selecting for cells containing the desired insertion. Several methods have been developed, one of which is shown in Fig. 1. In this example the gene is disrupted by a Tn5neo gene and flanked by a gene encoding thymidine kinase ( $E C$ 2.7.1.21; $t k$ gene). Cells expressing Tn5neo, which encodes the bacterial enzyme aminoglycoside $3^{\prime}$-phosphotransferase, are resistant to the antibiotic G418. Cells expressing the $t k$ gene are sensitive to treatment with the nucleotide analogue gancyclovir. Also shown in Fig. 1 is the outcome of a non-homologous recombination event; the DNA integrates randomly and the transfected cell is resistant to the antibiotic G418 and sensitive to gancyclovir. During homologous recombination (Fig. 1) the integrated transgene retains the Tn5neo gene but loses the $t k$ gene. The cells are both G418 and gancyclovir resistant, and can be selected by incubation in the presence of these reagents.

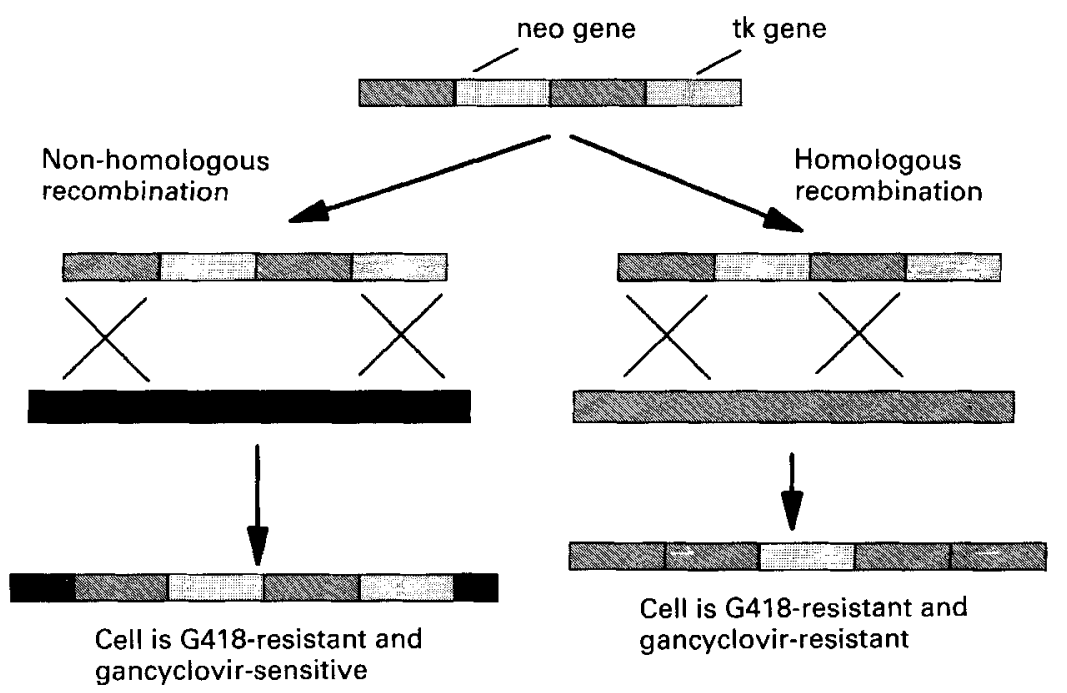

Fig. 1. Knocking-out genes by homologous recombination. Embryonic stem cells are transfected with the DNA construction similar to that shown at the top of the diagram. Integration of the gene at a specific site by homologous recombination renders the cell resistant to G418 and gancyclovir. These cells can be selected in culture and implanted into mice. $t k$ Gene, gene encoding thymidine kinase ( $E C$ 2.7.1.21). For further details, see text. The insertion of the neo gene within the coding region of the target gene renders the target inactive in the transgenic construct. 


\section{APPLICATIONS}

\section{Tissue-specific gene expression}

Transgenesis has been used to study mechanisms involved in tissue-specific aspects of gene expression, and also to identify genes that are expressed at a particular time and position during embryonic development.

The insulin gene provides an excellent example of tissue-specific aspects of gene expression. In the adult it is expressed exclusively in the beta cells of the islets of Langerhans, although some recent data show the presence of mRNA in some highlylocalized areas of the brain (Docherty \& Clark, 1995). In a classic experiment, Hanahan (1985) linked the insulin promoter to the SV40 large-T antigen (Tag), micro-injected this DNA construction into mouse embryos, and examined the expression of Tag in various tissues from the resultant offspring. The results demonstrated that a defined region of the insulin promoter was sufficient to restrict expression of the transgene to the pancreas. In further experiments specific mutations and deletions in the promoter region were made and their effects on the expression of the transgene in various tissues monitored (Fromont-Racine et al. 1990). These experiments helped to map regulatory sequences $5^{\prime}$ upstream of the insulin gene that were active only in the $\beta$ cells of the islets of Langerhans. Interestingly, the $\beta$ cells expressing the Tag developed tumours from which pancreatic $\beta$ cell lines could be derived (see p. 617).

To identify gene regulatory sequences that are active at a particular time and tissue during development, an enhancer trap technique has been developed (see Fig. 2; Kollias \& Grosveld, 1992). In this technique, a DNA construction is made in which the $\beta$-galactosidase (EC 3.2.1.23) gene is linked to a constitutive promoter. Transgenic mice which are generated using this construction are killed at certain stages during embryonic development and the tissues stained for $\beta$-galactosidase expression by immunocytochemistry. Expression of $\beta$-galactosidase is dependent on the transgene integrating close to an enhancer. Thus, $\beta$-galactosidase will only be expressed in a manner dependent on the temporal and spatial activity of the enhancer. Such enhancers can be mapped readily by cloning and sequencing fragments containing the transgene and adjacent DNA.

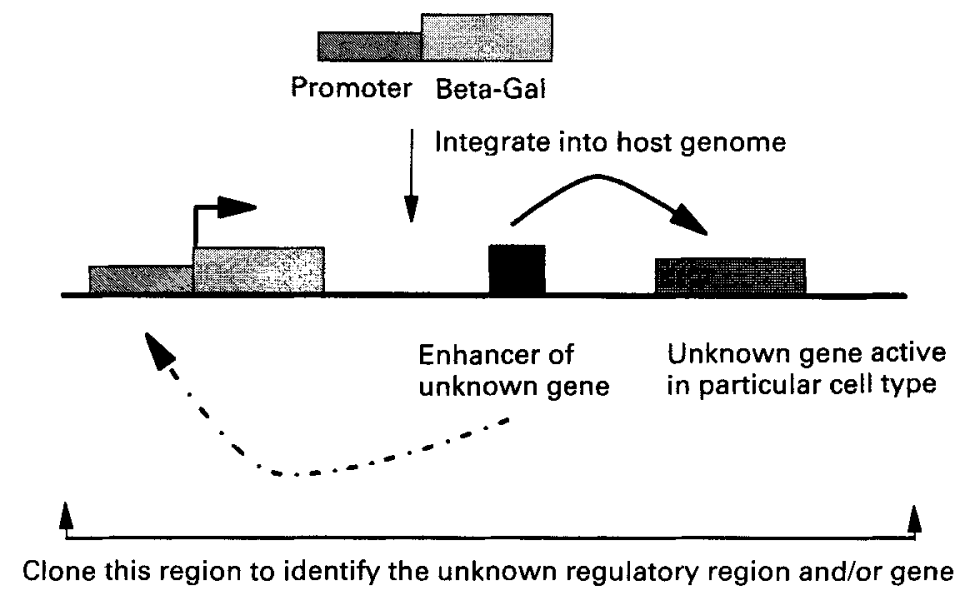

Fig. 2. An example of an enhancer trap that operates by distant transcription effects. Beta-Gal, $\beta$-galactosidase (EC 3.2.1.23) gene. For details of procedures used, see text. 


\section{Cell ablation}

Transgenic technology can be used to ablate specific cell types (Bernstein \& Breitman, 1989). The most commonly used method involves attaching a tissue-specific promoter to the gene encoding the A chain of ricin or the A chain of diptheria toxin (Palmiter et al. 1987). Thus, the promoters of the crystallin gene, elastin gene and growth hormone or prolactin genes have been linked to diptheria-toxin A-chain gene and introduced into transgenic mice to ablate the lens cells of the eye, the pancreas, and pituitary cells respectively. However, a problem with this approach is that if the toxin gene is switched on early in development, the ablation of particular cell types may be lethal. This can be surmounted by using an inducible system for selective killing which involves linking the tissue-specific promoter to the gene encoding thymidine kinase (TK). Expression of the $\imath k$ gene is not itself deleterious to the cell, but the viral enzyme, in contrast to mammalian TKK, can phosphorylate, and thereby convert a number of drugs such"ias acyclovir and gancyclovir to toxic products. Thus, these drugs can be injected into the mice at a particular time-point to induce ablation of specific actively-dividing cells expressing the herpes $t k$ gene (Heyman et al. 1985).

\section{Gene knock-outs}

Homologous recombination in embryonic stem cells has provided a method for inactivating (or knocking-out in the parlance of the molecular biologist) a particular gene. At present as few as $5 \%$ of the total number of genes in the human genome have so far been identified. As a result of the human genome project and related genetic studies it is inevitable that this percentage will increase dramatically over the next few years. There will be a requirement for functional studies that will describe the gene product in terms of its role in various cellular processes and its effect on whole animal physiology. One way of determining such functions will be to specifically inactivate the gene.

There are numerous examples of the use of knock-out experiments to prove that a gene has a particular function. Insulin promoter factor 1 (IPF1) is a recently cloned homeodomain transcription factor that is expressed almost exclusively in the pancreatic $\beta$ cells where it is thought to play an important role in insulin gene expression (Ohlsson et al. 1993). When the IPF1 gene was knocked-out in transgenic mice, it was found that the mice failed to develop a pancreas, confirming that this factor played a major role in cell commitment towards a particular pancreatic cell lineage (Jonsson et al. 1994).

Knock-out mice have been developed also as models for human disease. The cystic fibrosis mouse contains a defective CFTR gene, the MDX mouse contains a mutation in the mouse homologue of dystrophin, while mice harbouring a targeted mutation in the type 1 collagen gene have been used as a model for osteogenesis imperfecta. However, not all mouse gene mutations result in the same gene phenotype as seen in humans. Mice containing a targeted mutation in the hypoxanthine-guanine phosphoribosyl transferase (EC 2.4.2.8; HPRT) gene are genetically similar to patients with Lesch-Nyhan syndrome; however, these mice do not exhibit symptoms of the disease. This is because mice use urate oxidase ( $E C$ 1.7.3.3) as well as HPRT for the salvage of hypoxanthine and guanine, while humans only use HPRT. Lesch-Nyhan mouse models, therefore, would require a double knock-out of both these enzymes. Knock-out mouse models of disease have been used also in developing and testing approaches to gene therapy. For example, 
the shiverer mouse, which is deficient in myelin, has been treated by introducing a normal myelin basic protein gene into embryos (Readhead et al. 1987).

\section{Production of novel cell lines}

There is an ever-increasing demand for novel mammalian cell lines, not only for basic biological research, but also for their uses in the pharmaceutical industry for discovering novel therapeutics. Immortalization of cells usually involves interfering with the activity of tumour suppresser genes such as P53 and retinoblastoma (Rb). This is usually done by introducing into the cell and culture the viral oncogene, SV40Tag. SV40Tag acts by sequestering P53 and Rb, thus permitting the cell to be cultured indefinitely. Consequently, several lines have been developed by linking SV40Tag to tissue-specific promoters (such as insulin, see p. 615) and introducing these constructs as a transgene into mice. However, the problem with SV40Tag is that it still retains some transforming activity and the resulting cell lines tend to dedifferentiate. This has to some extent been surmounted by using temperature-sensitive mutants of SV40Tag; at raised temperatures $\left(39^{\circ}\right)$ the mutant (SV40tsTag) loses its ability to bind to P53 and Rb, while at lower temperatures $\left(33^{\circ}\right)$ it binds P53 and Rb (Jat \& Sharp, 1989). Cell lines generated from transgenic mice harbouring this temperature-sensitive variant of Tag may have widespread applications. For example, at the permissive temperature rat hepatocytes immortalized with SV40tsTag secrete low levels of albumin and transferrin, but the cells grow as an immortalized line. On switching to the non-permissive temperature, the secretion of albumin and transferrin is increased between 10- and 20-fold, thus emphasizing that the specialized functions of the liver only appear when functional SV40tsTag is removed from the cell (Zaret et al. 1988).

An alternative approach has been adopted by Efrat et al. (1995) to generate pancreatic $\beta$ cell lines for use in cell therapy for diabetes. This involved crossing two strains of transgenic mice, one harbouring a transgene containing Tag linked to the bacterial tetracycline (tet)-resistant operon regulatory system. This transgene was stably integrated into the mouse genome but not expressed (because it lacked an appropriate mammalian promoter). The second strain harboured a DNA construction containing a gene encoding the tet activator protein under the control of the insulin promoter, such that the tet activator was expressed in pancreatic $\beta$ cells. The offspring that resulted from these two strains expressed Tag in the $\beta$ cells under the control of the tet activator protein. However, when the cells were treated with a tetracycline analogue the activator protein no longer stimulated expression of Tag from the tet operator and the cells stopped growing. This type of genetic switch will have many uses in engineering mammalian cell lines for therapeutic purposes.

\section{Production of pharmaceutical proteins}

Transgenic animals, in particular sheep, have also been used for the production of human therapeutic proteins. This is based on the ability of the mammary gland to process and secrete large volumes of protein-rich fluid. The strategy adopted involves linking the gene encoding the protein to the regulatory region of one of several milk proteins. Using a bovine $\beta$ lactoglobulin promoter as much as $65 \mathrm{~g}$ human $\alpha 1$ antitrypsin/l has been produced in the milk of transgenic sheep (Carver et al. 1992). 


\section{CONCLUDING REMARKS}

During the past decade or so the use of transgenic animals has developed to the stage where it can now be regarded as an important if not essential facility within every biomedical research establishment. The rapid advances in gene cloning and sequencing have led to the appearance within various databases of a plethora of genes of unknown function. It is clear that a transgenic approach will be important in establishing a functional role for many of these sequences.

\section{REFERENCES}

Bernstein, A. \& Breitman, M. (1989). Genetic ablation in transgenic mice. Molecular Biology and Medicine 6 , $523-530$.

Capecchi, M. R. (1989). Altering the genome by homologous recombination. Science 244, 1288-1292.

Carver, A., Wright, G., Cottom, J., Cooper, M., Dalrymple, M., Temperley, S., Udell. M., Reeves, D., Percy, J., Scott, A., Barrass, D., Gibson, Y., Jeffrey, Y., Samuel, C., Colman, A. \& Garner, I. (1992). Expression of human $\alpha 1$ antitrypsin in transgenic sheep. Cytotechnology 9, 77-84.

Docherty, K. \& Clark, A. R. (1995). In Diabetes: Clinical Science in Practice, pp. 15-31 [R. D. G. Leslie and D. Robbins, editors]. Cambridge: Cambridge University Press.

Efrat, S.. Fusco-DeMane, D., Lemberg, H.. Al Emran, O. \& Wang, Z. (1995). Conditional transformation of a pancreatic $\beta$ cell line derived from transgenic mice expressing a tetracycline-regulated oncogene. Proceedings of the National Academy of Sciences, USA 92, 3567-3580.

Evans, M. J. \& Kaufman, M. H. (1981). Establishment in culture of pluropotential cells from mouse embryos. Nature 292, 154-156.

Fromont-Racine, M. , Bucchini, D., Madsen, O., Desbois, P., Linde, S., Nielsen, J. H., Saulnier, C., Ripoche, M.-A., Jami, J. \& Pictet, R. (1990). Effect of 5'-flanking sequence deletions on expression of the human insulin gene in transgenic mice. Molecular Endocrinology 4, 669-677.

Hanahan, D. (1985). Heritable formation of pancreatic $\beta$ tumours in transgenic mice expressing recombinant insulin/simian virus 40 oncogenes. Nature 315, 115-122.

Heyman, R. A., Borrelli, E., Lesley, J., Anderson, D., Richman, D. D., Baird, S. M., Heyman, R. \& Evans, R. M. (1989). Thymidine kinase obliteration: creation of transgenic mice with controlled immune deficiency. Proceedings of the National Academy of Sciences, USA 86, 2698-2702.

Jat, P. S. \& Sharp, P. A. (1989). Cell lines established by a temperature-sensitive simian virus-40 large T-antigen are growth restricted at the nonpermissive temperature. Molecular and Cell Biology 9. 1672-1681.

Jonsson. J., Carlsson, L., Edlund, T. \& Edlund, H. (1994). Insulin-promoter-factor 1 is required tor pancreas development in mice. Nature 371, 606-609.

Kollias, G. \& Grosveld, F. (1992). The study of gene regulation in transgenic mice. In Transgenic Animals, pp. 79-98 [F. Grosveld and G. Kollias, editors]. London and New York: Academic Press.

OhIsson, H., Karlsson, K. \& Edlund, T. (1993). IPF-1, a homeodomain-containing transactivator of the insulin gene. EMBO Journal 12, 4251-4259.

Palmiter, R. D., Behringer, R. R., Quaife, C. J., Maxwell, F., Maxwell, I. H. \& Brinster, R. L. (1987). Cell lineage ablation in transgenic mice by cell-specific expression of a toxin gene. Cell 50, 435-443.

Readhead, C., Popko, B., Takahashi, N., Shine, H. D., Saavedra, R. A., Sidman, R. L. \& Hood, L. (1987). Expression of a myelin basic protein gene in transgenic shiverer mice: correction of the dysmyelinating phenotype. Cell 48. 703-712.

Smithies, O., Koralewski, M. A., Song. E. Y. \& Kucherlapati, R. S. (1984). Homologous recombination with DNA inserted into mammalian cells. Cold Spring Harbor Symposium on Quantitative Biology 49, $230-234$.

Zaret, K. S., DiPersio, C. M., Jackson, D. A., Montigny, W. J. \& Weinstat, D. L. (1989). Conditional enhancement of liver specific gene transcription. Proceedings of the National Academy of Sciences, USA 85, $9076-9080$. 